\title{
Roots and prespective of Bergmann-Einstein scalar tensor theory: the unpublished paper
}

Joseph Kouneiher*

Université de Nice Sophia Antipolis

E-mail: joseph.kouneiher@unice.fr

In 1938 long time before Dicks, Branes and Jordan work on scalar-tensor theory, Einstein and Bergmann introduce the modern viewpoint in which a four-dimensional theory that coincides with Einstein-Maxwell theory at long distances is derived from a five dimensional theory with complete symmetry among all five dimensions. But then they drew back, modifying the theory in a way that spoiled the five-dimensional symmetry and looks contrived to modern readers. The reason was that the more symmetric version of the theory predicts the existence of a new long range field (a massless scalar field), a prediction which Einstein and Bergmann refuse to admit. We know today that, with their similar, but slightly different predictions for physical phenomena, scalar-tensor theories turned out to be the first significant challenge to Einstein's theory in over forty years. But as it turned out, standard Einstein theory fared better in these tests than any of the alternatives. In consequence, in the 1970's, interest in these alternative theories dropped dramatically. However, from the 1980's on, new discoveries and theories have led to renewed interest in scalar-tensor gravity.

In this lecture I'll back to Begmann-Einstein paper and explain the issue of their drew back and the epistemological statut of the scalar tensor kind theories with respect to the last observations.

Frontiers of Fundamental Physics 14 - FFP14,

15-18 July 2014

Aix Marseille University (AMU) Saint-Charles Campus, Marseille

\footnotetext{
${ }^{*}$ Speaker.
} 